\title{
Estimation of teleported and gained parameters in a non-inertial frame N. Metwally \\ ${ }^{1}$ Department of Mathematics, College of Science, Bahrain University, Bahrain ${ }^{2}$ Department of Mathematics, Faculty of Science, Aswan University, Aswan, Egypt
}

\begin{abstract}
We estimate the teleported and the gained parameters by means of Fisher information in a non-inertial frame. The sender and the receiver share an accelerated maximum or partial entangled state. The estimation degree of these parameters depends on Unruh acceleration, the used single mode approximation(within/ beyond), the structure of the initial teleported state, and the entanglement of the initial communication channel. The maximization and minimization estimation degree of the teleported parameters depend on the initial encoded information. The estimation degree of the parameters can be maximized if the partners teleport classical information.
\end{abstract}

keywords: Estimation, Fisher information, Unruh acceleration

\section{Introduction}

In the context of the classical estimation theory, Fisher information represents a key value that used to estimate any parameter [1, 2]. Similarly, quantum Fisher information can be used as a measure of estimation in quantum information theory [3]. As an example, the optimal parameter estimation of Pauli channels is discussed by Ruppert et. al., [4. Q. Zheng et. al, [5] have used Fisher information to estimate the channel parameter of a two- qubit state, where each qubit interacts independently with its own environment. The possibility of estimating multi-quantum parameters is discussed by P. Yue et. al, [6].

Due to its important in estimation theory, there are many studies that have quantified the Fisher information for different models. For example, quantum Fisher information of the GHZ state in decoherence channel is quantified by J. Ma et. al [7]. The relation between the fidelity susceptibility and quantum Fisher information is investigated by J. Liu et. al [8. The dynamic of the quantum Fisher information in the Ising model is discussed in [9]. A. Altintas [10] studied the dynamics of quantum Fisher information of a steady state in a noisy environment. Recently, J. He et.al, [11 studied the possibility of enhancing the quantum Fisher information by using the uncollapsing measurements. Xiao et. al [12] proposed a scheme to enhance the teleported quantum Fisher information by utilizing partial measurements.

However, there are limited studies have been done in the context of non-inertial frame. For example, Yao et. al, [13] have investigated the performance of the quantum Fisher information under the Unruh-Hawking effect. The dynamics of Fisher information and skew information for Unruh effect within and witout external noise is discussed by Banerjee et. al [14]. Recently, Metwally [15] discussed the effect of the Unruh acceleration on the Fisher information for different classes of maximum and partial entangled state.

Therefore, we are motivated to discuss the possibility of estimating the teleported parameters by means of teleporting a quantum state between two users. In this proposal, it is assumed that one user (Alice) stays in inertial frame, while the second user (Bob) is accelerated with a uniform acceleration. 
The manuscript is organized as follows: in Sec.2, we describe different types of the initial states that Alice and Bob share, the relation between the Minkowski and Rindler spaces is reviewed, and the dynamics of the final state when only Bob's qubit is accelerated uniformly is obtained analytically. This state is used as a quantum communication channel to teleport unknown state from Alice to Bob as described in Sec.3. In Sec.4, we estimate the teleported parameters by means of Fisher information. Finally, we summarize our results in Sec.(5).

\section{The suggested Proposal}

We assume that, Alice and Bob as partners share a state prepared initially in a two qubit system of Bell, or $X$-state. It is considered that, only one qubit say, Alice's qubit is at a rest, while Bob's qubit is accelerated with a uniform Unruch acceleration. Alice's task is teleporting unknown state to Bob by using Bennett's protocol [16, 17]. Bob will use this teleported state to estimate the initial parameters of the teleported state and the gained parameters during the teleportation process. Bob will then quantify the Fisher information [3] corresponding to these parameters. In this context, we aim to investigate the effect of the Unruch acceleration, and the initial state settings of the communication channel between the partners, on the precision of estimation.

Let the partners, Alice and Bob share a self-transposed class of two qubits [18] as,

$$
\rho_{12}=\frac{1}{4}\left(I_{4 \times 4}+\sum_{i, j} \vec{\sigma}_{i}^{(1)} \cdot{ }^{\downarrow} C \cdot \vec{\sigma}_{j}^{(2)}\right),
$$

where $\vec{\sigma}^{(i)}=\left(\sigma_{x}^{(i)}, \sigma_{y}^{(i)}, \sigma_{z}^{(i)}\right)$, are the Pauli matrices of Alice's and Bob's qubits, respectively. The dyadic ${ }^{\triangleright} \vec{C}$ is a $3 \times 3$ matrix, where its elements are defined as $c_{i j}=\operatorname{tr}\left\{\rho_{12} \sigma_{i}^{(1)} \sigma_{j}^{(2)}\right\}$ [18]. From the state (10), different important states can be considered. However if we set $c_{11} \neq c_{22} \neq c_{33} \neq 0$ and $c_{i j}=0, i \neq j$ one obtains what is called $X$ - state. Moreover, if $c_{11}=c_{22}=c_{33}=-1$, and $c_{i j}=0, i \neq j$ one gets a maximum Bell state, singlet state $\rho_{\psi^{-}}$. Also, what is called Werner state can be obtained if $c_{11}=c_{22}=c_{33}=-F$ and $c_{i j}=0, i \neq j$. The dynamics of all these states in the non-inertial frame is discussed by Metwally [19].

\section{$2.1 \quad$ Unruh effect}

It has been shown that, for the perspective inertial observers the Minkowski coordinates are the most suitable choice to describe the Dirac qubit. On the other hand, from the scenery of the non-inertial observers, the Rindler coordinates are the most adequate coordinates to describe the Dirac qubits. Now, we assume that, Alice's qubit moves in the inertial frame, and described by Minkowski coordinates $(t, z)$, while Bob's qubit is uniformly accelerated with a constant acceleration $a$. Therefore, Bob's qubit can be described by using Rindler coordinates $(\tau, \eta)$, such that, $\tau=\tanh ^{-1}(t / x), \eta=\sqrt{x^{2}-t^{2}}, 0<\eta<\infty,-\infty<\tau<\infty$. These transformations define two regions in the space-time; the first region, $I$ for $|t|<z$ and the second region, $I I$ for $z<-|t|$. The accelerated qubit moves on a parabola in the first region $I$ defined by $\eta=1 / a, a$ is the uniform acceleration, while the Anti-accelerated qubit moves on the parabola $\eta=-1 / a$ in the second region $I I$ [20, 21]. To describe a Minkowski sate in terms of Rindler's space, one has to use the Bogoliubov transformation,

$$
\nu_{k}=\cos r \mathcal{C}_{k}^{I}-e^{-i \phi} \sin r \mathcal{D}_{k}^{I I}, \quad \mu_{k}^{\dagger}=e^{i \phi} \sin r \mathcal{C}_{k}^{I}+\cos r \mathcal{D}_{k}^{I I},
$$


where $\nu_{k}$ and $\mu_{k}^{\dagger}$ represent the annihilation and criterion operators in Minkowski space such that [20],

$$
\nu_{k}\left|0_{k}^{+}\right\rangle_{M}=0, \quad \mu_{k}\left|0_{k}^{-}\right\rangle_{M}=0, \quad \nu_{k}^{\dagger}\left|0_{k}^{+}\right\rangle_{M}=\left|1_{k}^{+}\right\rangle, \quad \mu_{k}^{\dagger}\left|0_{k}^{-}\right\rangle_{M}=\left|1_{k}^{-}\right\rangle_{M},
$$

and, $\mathcal{C}_{k}^{I}$ and $\mathcal{D}_{-k}^{I I^{\dagger}}$ represent the annihilation and creation operators in the regions $I$ and $I I$, respectively. The parameter $\phi$ is an unimportant phase that can be absorbed into the definition of the operators.

$$
\begin{aligned}
\left|0_{k}\right\rangle & =\cos r_{b}\left|0_{k}^{+}\right\rangle_{I}\left|0_{k}^{-}\right\rangle_{I I}+\sin r_{b}\left|1_{k}^{+}\right\rangle_{I}\left|1_{k}^{-}\right\rangle_{I I}, \\
\left|1_{k}\right\rangle & =q_{R}\left|1_{k}^{+}\right\rangle_{I}\left|0_{k}^{-}\right\rangle_{I I}+q_{L}\left|0_{k}^{+}\right\rangle_{I}\left|1_{k}^{-}\right\rangle_{I I},
\end{aligned}
$$

where $q_{R}$ and $q_{L}$ are complex numbers with $\left|q_{R}\right|^{2}+\left|q_{L}\right|^{2}=1$, and the dimensionless parameter $r$ is given from tanr $=e^{-\pi \omega \frac{c}{a}}, a \in[0, \infty), r \in[0, \pi / 4], \omega$ is the frequency of the travelling qubits, and $c$ is the speed of light. The transformation (44) mixes a particle (fermions) in region $I$ and an anti particle (anti-fermions) in region $I I$. In the computational basis $\left|0_{k}\right\rangle$ and $\left|1_{k}\right\rangle$ can be written as [21, 22].

\subsection{Accelerating the communication quantum state}

Let us consider that, the partners Alice and Bob share a state defined by (11). As described above, Alice's qubit remains stationary, while Bob's qubit is accelerated with a uniform acceleration. Bob is causally disconnected from the second region, therefore all the accessible information is encoded in Alice's and Bob's qubit in the first region. Consequently, if we trace out all the modes in the second region, the final state between Alice and Bob is given by,

$$
\begin{aligned}
\rho_{a b}^{a c c}= & \mathcal{B}_{1}|00\rangle\left\langle 00\left|+\mathcal{B}_{2}\right| 00\right\rangle\left\langle 11\left|+\mathcal{B}_{3}\right| 11\right\rangle\left\langle 00\left|+\mathcal{B}_{4}\right| 01\right\rangle\langle 01| \\
& +\mathcal{B}_{5}|10\rangle\left\langle 10\left|+\mathcal{B}_{6}\right| 10\right\rangle\left\langle 01\left|+\mathcal{B}_{7}\right| 01\right\rangle\left\langle 10\left|+\mathcal{B}_{8}\right| 11\right\rangle\langle 11|,
\end{aligned}
$$

where,

$$
\begin{aligned}
& \mathcal{B}_{1}=\mathcal{A}_{1} \cos ^{2} r+\mathcal{A}_{2}\left|q_{L}\right|^{2}, \quad \mathcal{B}_{2}=\mathcal{A}_{4} q_{R}^{*} \cos r+\mathcal{A}_{3} q_{L} \sin r, \\
& \mathcal{B}_{3}=\mathcal{A}_{3} q_{L}^{*} \sin r+\mathcal{A}_{4} q_{R} \cos r, \quad \mathcal{B}_{4}=\mathcal{A}_{1} \sin ^{2} r+\mathcal{A}_{2}\left|q_{R}\right|^{2}, \\
& \mathcal{B}_{5}=\mathcal{A}_{2} \cos ^{2} r+\mathcal{A}_{1}\left|q_{L}\right|^{2}, \quad \mathcal{B}_{6}=\mathcal{A}_{3} q_{R}^{*} \cos r+\mathcal{A}_{4} q_{L} \sin r, \\
& \mathcal{B}_{7}=\mathcal{A}_{3} q_{L}^{*} \sin r+\mathcal{A}_{3} q_{R} \cos r, \quad \mathcal{B}_{8}=\mathcal{A}_{2} \sin ^{2} r+\mathcal{A}_{1}\left|q_{R}\right|^{2},
\end{aligned}
$$

and $\left|q_{r}\right|^{2}+\left|q_{L}\right|^{2}=1, \mathcal{A}_{1}=\frac{1+c_{33}}{4}, \mathcal{A}_{2}=\frac{1-c_{33}}{4}, \mathcal{A}_{3}=\frac{c_{11}+c_{22}}{4}$ and $\mathcal{A}_{4}=\frac{c_{11}-c_{22}}{4}$.

In the next section, Alice and Bob will use the state (3) as a communication channel to teleport an unknown state from Alice to Bob by using Bennett protocol [16].

\section{Quantum Teleportation}

Now, the partners share the accelerated state(5) and Alice is asked to send the unknown state, $\rho_{u}$, to Bob where,

$$
\rho_{u}=|\alpha|^{2}|0\rangle\left\langle 0\left|+\alpha \beta^{*}\right| 0\right\rangle\left\langle 1\left|+\beta \alpha^{*}\right| 1\right\rangle\left\langle\left. 0|+| \beta\right|^{2} \mid 1\right\rangle\left\langle\left. 1|, \quad| \alpha\right|^{2}+|\beta|^{2}=1 .\right.
$$

Alice and Bob perform the teleporation protocol[16] by using the following steps. 
1. Alice performs a CNOT operation between her own qubit and the given one followed by the Hadamard gate on the given qubit.

2. Alice performs measurements on the two qubits on her hand and send her results to Bob via classical communication channel.

3. According to the received results, Bob performs the required operations to get the teleported state.

Alice and Bob perform the steps(1-3) to teleport the unknown state $\rho_{u}$. Finally, if Alice measures 00, then Bob will obtain the state,

$$
\rho_{B o b}=\varrho_{00}|0\rangle\left\langle 0\left|+\varrho_{01}\right| 0\right\rangle\left\langle 1\left|+\varrho_{10}\right| 1\right\rangle\left\langle 0\left|+\varrho_{11}\right| 1\right\rangle\langle 1|,
$$

where,

$$
\begin{array}{rlrl}
\varrho_{00} & =\frac{1}{2}\left(|\alpha|^{2} \mathcal{B}_{1}+|\beta|^{2} \mathcal{B}_{5}\right), & \varrho_{01} & =\frac{1}{2}\left(\alpha \beta^{*} \mathcal{B}_{2}+\beta \alpha^{*} \mathcal{B}_{6}\right), \\
\varrho_{10}=\frac{1}{2}\left(\alpha \beta^{*} \mathcal{B}_{7}+\beta \alpha^{*} \mathcal{B}_{3}\right), & \varrho_{11} & =\frac{1}{2}\left(|\alpha|^{2} \mathcal{B}_{4}+|\beta|^{2} \mathcal{B}_{8}\right) .
\end{array}
$$

Let us assume that, the coefficients $\alpha=\cos (\theta / 2)$ and $\beta=\sin (\theta / 2) e^{i \phi}$, where the parameters $\theta \in[0, \pi]$ and $\phi \in[0,2 \pi]$ are the weight and the phase angles, respectively. Then (9) can be written explicitly as,

$$
\begin{aligned}
\varrho_{00} & =\frac{1}{8}\left[\cos ^{2} r\left(1+c_{33} \cos \theta\right)+\left|q_{L}\right|^{2}\left(1-c_{33} \cos \theta\right)\right], \\
\varrho_{01} & =\frac{\sin \theta}{8}\left[c_{11} \cos \phi\left(q_{R}^{*} \cos r+q_{L} \sin r\right)+i c_{22} \sin \phi\left(q_{R}^{*} \cos r-q_{L} \sin r\right)\right], \\
\varrho_{01} & =\frac{\sin \theta}{8}\left[c_{11} \cos \phi\left(q_{L}^{*} \sin r+q_{R} \cos r\right)+i c_{22} \sin \phi\left(q_{L}^{*} \sin r-q_{R} \cos r\right)\right], \\
\varrho_{11} & =\frac{1}{8}\left[\sin ^{2} r\left(1+c_{33} \cos \theta\right)+\left|q_{R}\right|^{2}\left(1-c_{33} \cos \theta\right)\right] .
\end{aligned}
$$

Now, we have all details to estimate the weight $(\theta)$, the phase $(\phi)$ and the Unruh $(r)$ parameters by calculating the Fisher information corresponding to each parameter as we see in the next sections.

\section{Fisher Information}

It is clear that, the final teleported state depends on the initial parameters, the weight and the phase parameters as well as the Unruh parameter which is gained during the teleportation process. The main task of the following subsections is estimating these parameters by calculating the Fisher information corresponding to these parameters.

It is well known that, any single mixed qubit can be described by its Bloch vector as,

$$
\rho=\frac{1}{2}\left(1+\vec{s} \cdot \sigma^{\downarrow}\right)
$$


where $\vec{s}=\left(s_{x}, s_{y}, s_{z}\right)$ and $\sigma^{\downarrow}=\left(\sigma_{x}, \sigma_{y}, \sigma_{z}\right)^{T}$. Fisher information for a mixed state with respect to a parameter $\kappa$, which will be estimated, can be described by means of the Bloch vector as [23],

$$
\mathcal{F}_{\kappa}=\left|\frac{\partial \vec{s}}{\partial \kappa}\right|^{2}+\frac{1}{1-|\vec{s}|^{2}}\left(\vec{s} \cdot \frac{\partial \vec{s}}{\partial \kappa}\right)^{2}
$$

while for pure state, namely $|\vec{s}|=1$, the Fisher information $\mathcal{F}_{\kappa}=\left|\frac{\partial \vec{s}}{\partial \kappa}\right|^{2}$.

Now, to quantify the amount of the teleported Fisher information which contained in the state (8), we describe it by means of its Bloch vector as,

$$
\rho_{\text {Bob }}=\frac{1}{2}\left(1+s_{x} \sigma_{x}+s_{y} \sigma_{y}+s_{z} \sigma_{z}\right)
$$

where $s_{i}=\operatorname{Tr}\left\{\rho_{B o b} \sigma_{i}\right\}, i=x, y$ and $z$,

$$
\begin{aligned}
s_{x}= & \frac{\sin \theta}{8}\left[c_{11} \cos \phi\left\{\left(q_{R}^{*}+q_{R}\right) \cos r+\left(q_{L}^{*}+q_{L}\right) \sin r\right\}\right. \\
& \left.+i c_{22} \sin \phi\left\{\left(q_{R}^{*}-q_{R}\right) \cos r+\left(q_{L}^{*}-q_{L}\right) \sin r\right\}\right] \\
s_{y}= & \frac{\sin \theta}{8}\left[c_{22} \sin \phi\left\{\left(q_{R}^{*}+q_{R}\right) \cos r-\left(q_{L}^{*}+q_{L}\right) \sin r\right\}\right. \\
& \left.+i c_{11} \cos \phi\left\{\left(q_{R}-q_{R}^{*}\right) \cos r+\left(q_{L}-q_{L}^{*}\right) \sin r\right\}\right] \\
s_{z}= & \frac{1}{8}\left[\cos 2 r\left(1+c_{33} \cos \theta\right)+\left(\left|q_{L}\right|^{2}-\left|q_{R}\right|^{2}\right)\left(1-c_{33} \cos \theta\right)\right] .
\end{aligned}
$$

\subsection{Estimation of the weight parameter, $\theta$}

In this investigation, we assume that the partners initially share maximum entangled state of Bell type, $\rho_{\phi^{+}}$or $\rho_{\psi^{-}}$, partial entangled state of $X$-state. In this context, we shall estimate the teleported weight and phase parameters by calculating the Fisher information with respect to these two parameters.

Fig.(1) displays the behavior of the Fisher information $\mathcal{F}_{\theta}(\theta, r)$ at fixed $\phi=\pi / 4$ with respect to the weight parameter, $\theta$ of the teleported state (13) and the Unruh acceleration within and beyond the single approximation. In Fig.(1a), we consider $q_{R}=1$ while $q_{L}=0$, namely within the single mode approximation, (WSMA). The general behavior shows that, Fisher information decreases as $r$ increases. The effect of the weight parameter $\theta$ appears clearly for large values of $r$, where $\mathcal{F}_{\theta}(\theta, r)$ decreases as $r$ increases to reach its minimum values at $\theta=\pi / 2$. However, for further values of $\theta$, the Fisher information increases gradually to reach its maximum value at $\theta=\pi$.

This behavior changes dramatically when we consider the Unruh effect beyond the single mode approximation,(BSMA), namely the single qubit has right and left components, where we set $q_{R}=q_{L}=1 / \sqrt{2}$. As it is described in Fig.(1b), Fisher information increases as $r$ increases. Moreover, as $\theta$ increases, Fisher information decreases to reach its minimum values at $\pi / 2$. Then as the weight parameter increases, $\mathcal{F}_{\theta}(\theta, r)$ increases gradually to reach its maximum values. These maximum values depend on the Unruh acceleration, where the maximization of $\mathcal{F}_{\theta}(\theta, r)$ is depicted at large values of $r$.

The effect of the phase parameter, $\phi$ on the dynamics of Fisher information, $\mathcal{F}_{\theta}(\theta, \phi)$ at fixed $r=\pi / 8$ is depicted in $\operatorname{Fig}(2)$. The behavior of the Fisher information within the 

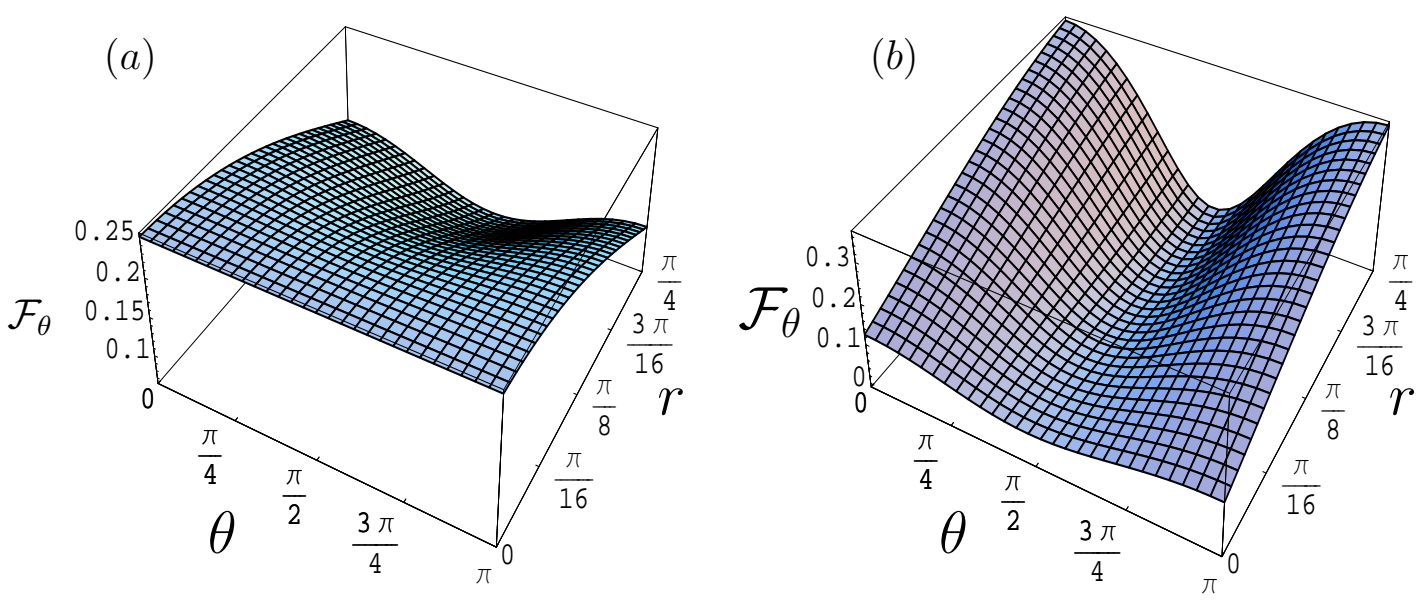

Figure 1: The dynamics of Fisher information $\mathcal{F}_{\theta}(\theta, r)$ at a fixed $\phi=\pi / 4$ against the Unruh parameter, $r$ : (a) WSMA, namely, $q_{R}=1$ and $q_{L}=0$, and (b)BSMA with $q_{R}=q_{L}=1 / \sqrt{2}$.
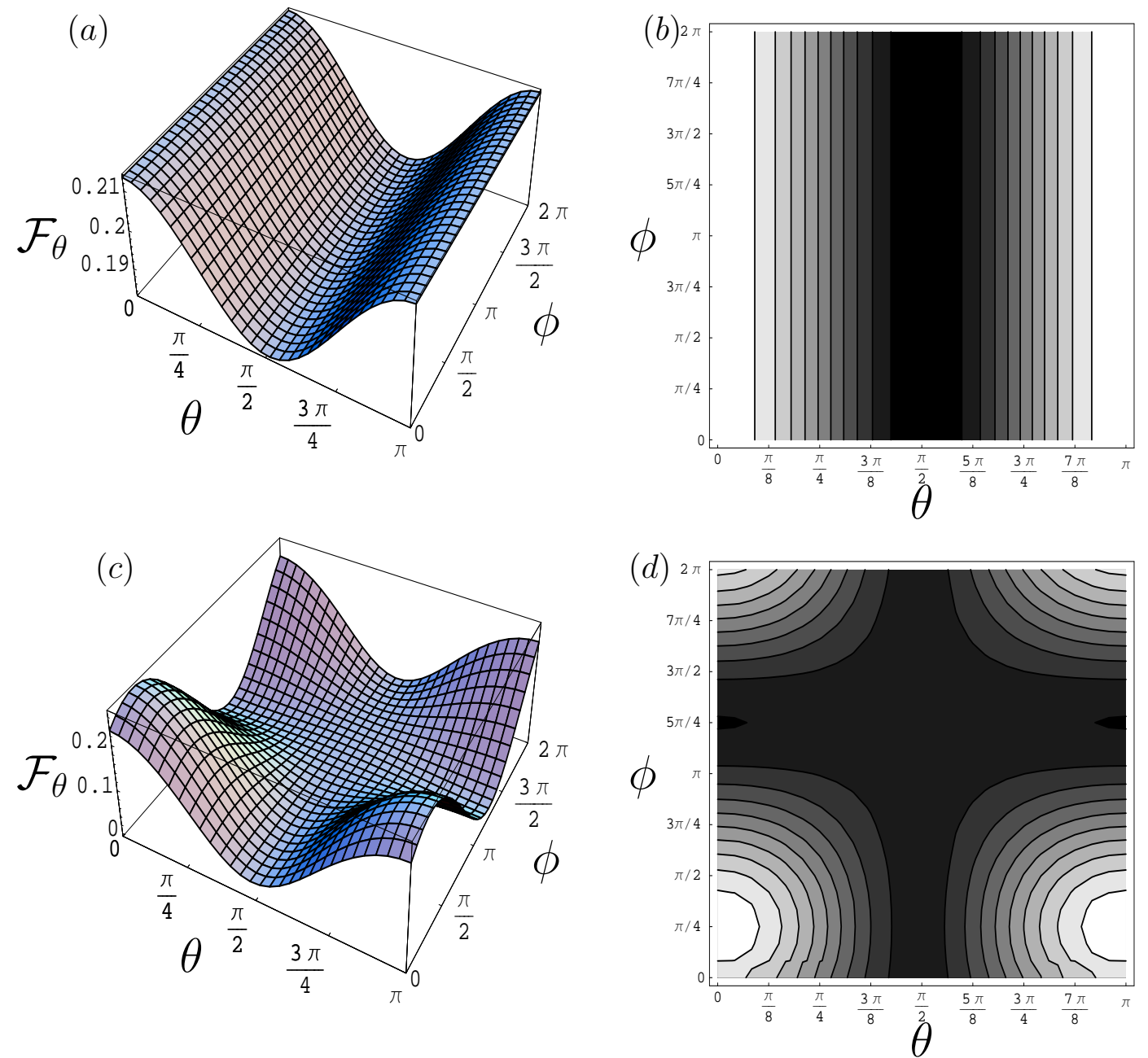

Figure 2: The same as Fig.(1) but it represents the Fisher information, $\mathcal{F}_{\theta}(\theta, \phi)$ at a fixed $r=\pi / 8$ is plotted against the phase parameter $\phi$. 
single mode approximation is displayed in Fig.(2a). It is clear that, the phase parameter has a negligible effect on the behavior of $\mathcal{F}_{\theta}(\theta, \phi)$. On the other hand, the phenomena of the sudden decay of the Fisher information is displayed as soon as $\theta$ increases. However, at $\theta \simeq \pi / 2, \mathcal{F}_{\theta}(\theta, \phi)$ vanishes completely. For further values, Fisher information re-birth again to reach its maximum value at $\theta=\pi$. Fig. $(2 \mathrm{~b})$ shows the behavior of $\mathcal{F}_{\theta}(\theta, \phi)$, but in a contour description, where it displays clearly, the values of $\theta$ at which Fisher information decreases/increases and the values of $\theta$ that maximize or minimize $\mathcal{F}_{\theta}(\theta, \phi)$.

Fig. (2c) displays the behavior of Fisher information $\mathcal{F}_{\theta}(\theta, \phi)$ against the phase parameter, $\phi$ BSMA, where we fixed the value of Unruh acceleration, $r=\pi / 8$. In this case, the phase parameter has a completely different effect. It is evident that, as $\phi$ increases, $\mathcal{F}_{\theta}(\theta, \phi)$ decreases gradually to reach its minimum values at $\phi=\pi$. For further values of $\phi$, the Fisher information completely vanishes to re-birth again for $\phi \in[3 \pi / 4,2 \pi]$. These results are displayed in Fig. $(2 \mathrm{~d})$, where the values of $\theta$ and $\phi$ which maximize and minimize $\mathcal{F}_{\theta}$ are seen clearly.

Figs. $(3 a \& 3 b)$ are devoted to investigate the effect of the phase parameter, $\phi$ on the Fisher information, $\mathcal{F}_{\theta}(\theta, \phi)$ BSMA at fixed $r=\pi / 8$, where the partners initial share the singled state, $\rho_{\psi^{-}}$. It is evident that, $\mathcal{F}_{\theta}(\theta, \phi)$ increases as $\phi$ increases in the interval $[0, \pi]$ and reaches its maximum values at $\phi=3 \pi / 4$, then it decreases again at further values of $\phi$ to vanish completely at $\phi \in[3 \pi / 2,2 \pi]$. On the other hand, as $\theta$ increases, $\mathcal{F}_{\theta}(\theta, \phi)$, decreases gradually to vanish completely at $\theta \in[\pi / 4,3 \pi / 4]$. For further values of $\theta, \mathcal{F}_{\theta}(\theta, \phi)$ re-births again to reach its maximum bounds at $\theta=\pi$.

Figs. $(3 c \& 3 d)$ display the behavior of $\mathcal{F}_{\theta}(\theta, \phi)$ WSMA for a system is initially prepared in the $X$-state, where we set $c_{11}=-0.9, c_{22}=-0.8, c_{33}=-0.7$. In this case, the effect of phase parameter, $\phi$ is different from that depicted in Fig. $(3 a \& 3 b)$, where $\mathcal{F}_{\theta}(\theta, \phi)$ reaches its maximum values at $\phi=0, \pi, 2 \pi$. Moreover, $\mathcal{F}_{\theta}(\theta, \phi)$ doesn't vanish completely for any value of $\phi \in[0,2 \pi]$. The vanishing phenomena of the Fisher information is due to the weight parameter, where it decayes suddenly as $\theta$ increases. From Fig. $(3 d)$, it is clear that $\mathcal{F}_{\theta}(\theta, \phi)$ vanishes completely at $\theta \in[3 \pi / 8,5 \pi / 8]$. For further values of $\theta$, Fisher information re-birthes again to reach its maximum bounds at $\theta=\pi$.

From these Figs.(1-3), one concludes that, the entanglement of the initial communication channel between the users play an important role on the teleported amount of Fisher information, where the upper bounds of the teleported Fisher information is large if the users use initially a maximum entangled communication channel. The phenomena of the sudden decay of Fisher information is depicted WSMA as Unruh acceleration increases. Moreover, the sudden increasing pheneomena of the Fisher information is displayed for large values of Unruh acceleration BSMA, while the gradually increasing behavior is displayed BSMA.

Moreover, the degree of estimating the teleported weight parameter $\theta$, depends on the type of information which is encoded on the teleported state. It is clear that, at $\theta=0$ or $\pi, \mathcal{F}_{\theta}(\theta, \phi)$ is maximum, where at these values the initial teleported state reduces to be $|\psi\rangle=|0\rangle$ and $|\psi\rangle=e^{i \phi}|1\rangle$, respectively, which means that the state carries only classical information. On the other hand, the minimum values of the estimation degree appears at $\theta=\pi / 2$, where the initial teleported state is defined by $|\psi\rangle=\frac{1}{\sqrt{2}}\left(|0\rangle \mp e^{i \phi}|1\rangle\right)$, which means that the initial state carries quantum information. 

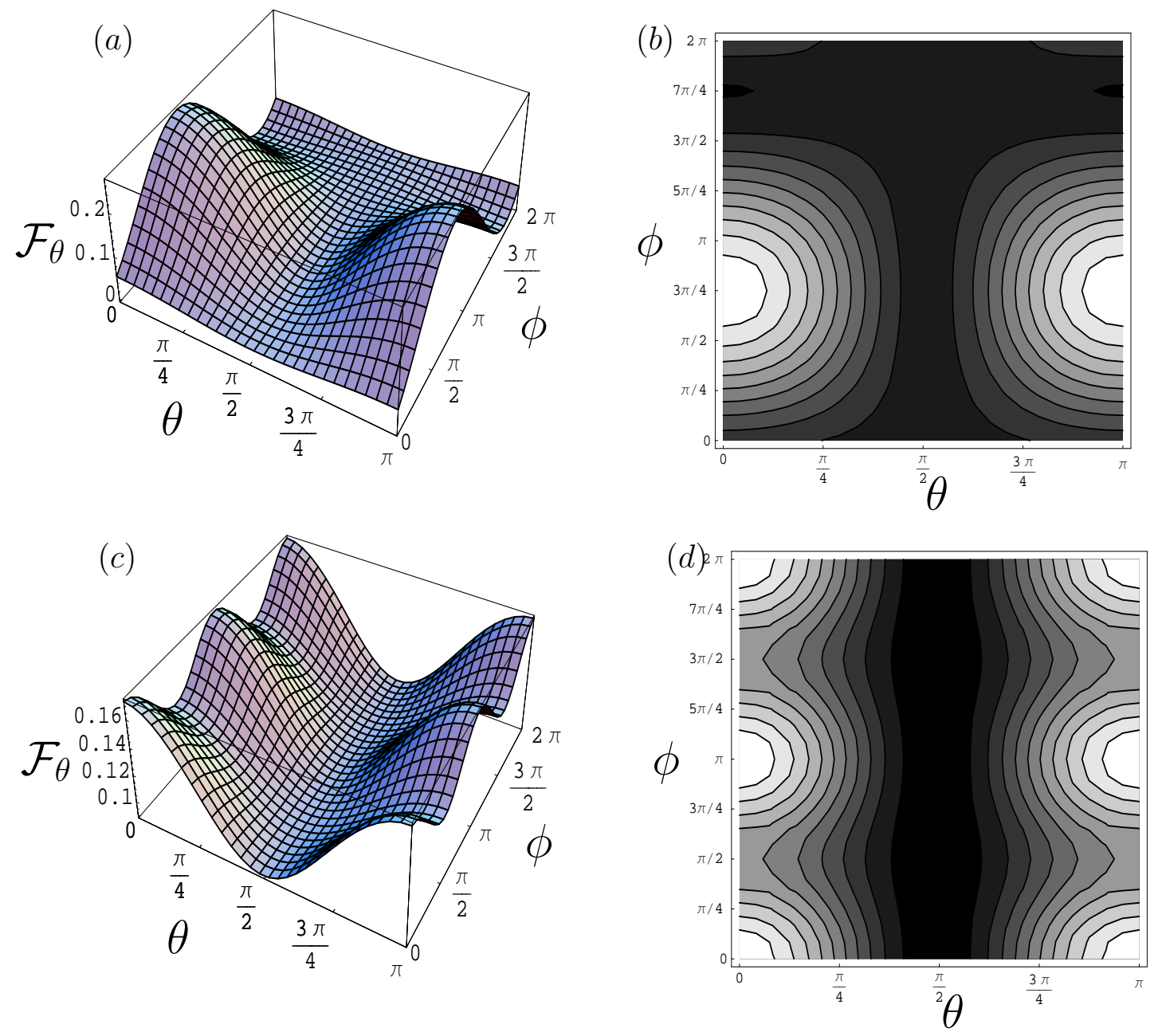

Figure 3: (a)The Fisher information, $\mathcal{F}_{\theta}(\theta, \phi)$ at fixed $r=\pi / 8$, BSMA for system is prepared initially in $\rho_{\psi^{-}}$, (b) WSMA for system is prepared initially in $X$-state with $c_{11}=$ $-0.9, c_{22}=-0.8, c_{33}=-0.7$.

\subsection{Estimating the phase parameter, $\phi$}

The behavior of Fisher information, $\mathcal{F}_{\phi}(\phi, r)$ at a fixed value $\theta=\pi / 4$ with respect to the phase parameter is shown in Fig.(4). Figs.(4a) and (4b) display the effect of the Unruh acceleration on the teleported Fisher information within/beyond the single mode approximation, respectively. The general behavior shows that, $\mathcal{F}_{\phi}(\phi, r)$ decreases as $r$ increases. The phenomena of the sudden changes of Fisher information appears within/beyond the single mode approximation. Within the single mode approximation, the upper bounds of the Fisher information $\mathcal{F}_{\phi}(\phi, r)$ is larger than that depicted for the beyond single mode approximation.

Figs. $(4 c \& 4 d)$ show the behavior of $\mathcal{F}_{\phi}(\phi, \theta)$ at a fixed value of the Unruh acceleration, $r=\pi / 8$, within/beyond the single mode approximation, respectively. In Fig (4c), the weight parameter's effect decreases as $\phi$ increases. The maximum values of $\mathcal{F}_{\phi}(\phi, \theta)$ are depicted at $\phi=\pi$ and $2 \pi$, while the weight parameter can be arbitrary.

This effect is dramatically changed in the presence of the BSMA. It is evident that, at small values of $\phi, \mathcal{F}_{\phi}(\phi, \theta)$, decreases as $\theta$ increases to reach its minimum value for the first 

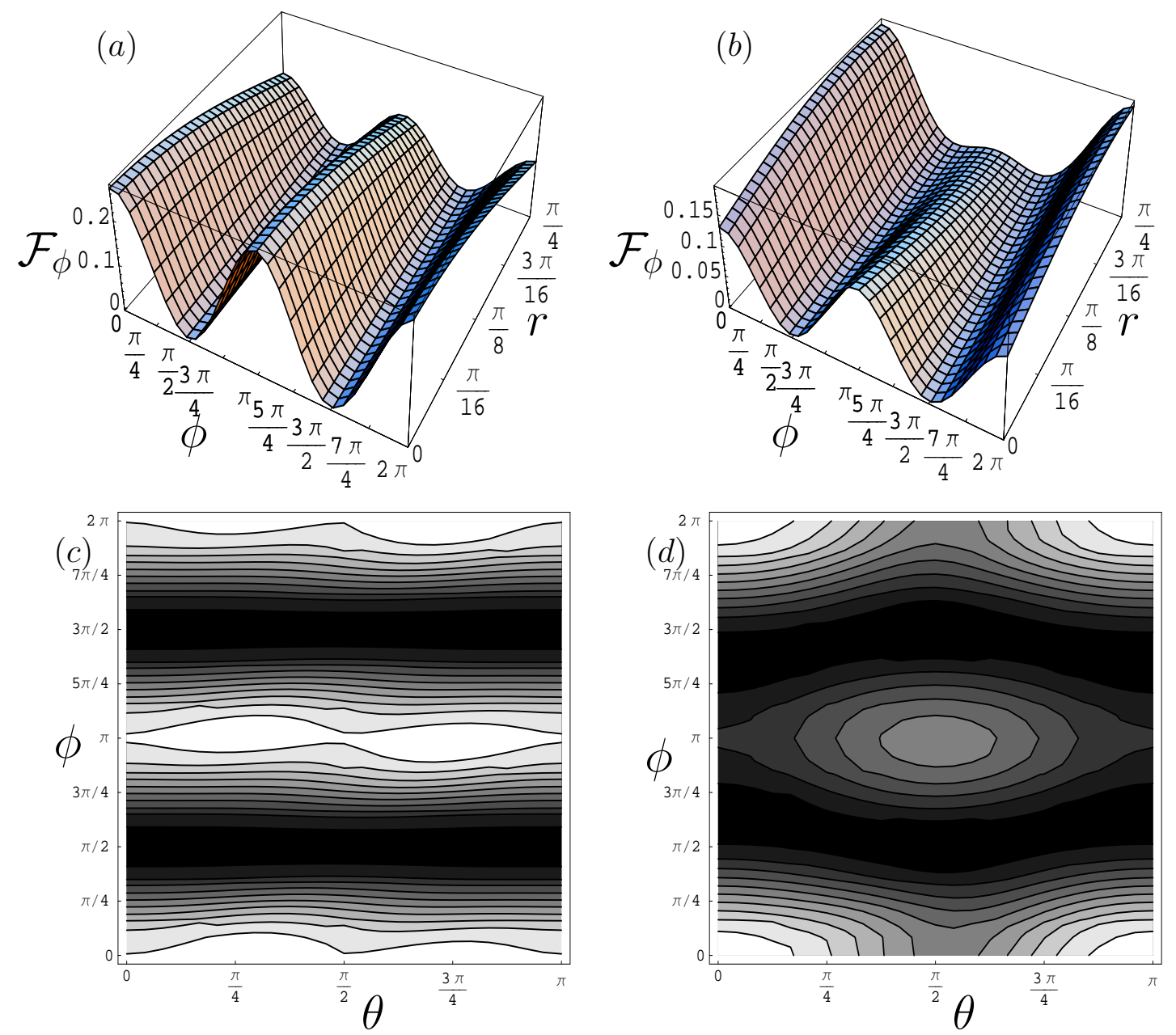

Figure 4: The teleported Fisher information $\left.\mathcal{F}_{\phi} \phi, r\right)$, at fixed $\theta=\pi / 4$ with respect to the parameter $\phi$, for (a) WSMA i.e., $q_{L}=1$, and (b) BSMA, with $q_{L}=q_{r}=\frac{1}{\sqrt{2}}$. The Fisher information $\mathcal{F}_{\phi}(\phi, \theta)$ at fixed $r=\pi / 8$ for (c)WSMA and (b) BSMA.

time at $\theta=\pi / 2$. For further values of $\theta, \mathcal{F}_{\phi}(\phi, \theta)$ increases gradually to reach its maximum values at $\theta=\pi$. The effect of the weight parameter is completely disappear as $\phi$ increases. However, as $\phi$ increases in the interval $[\pi / 2,3 \pi / 4]$, and arbitrary values of $\theta$, the Fisher information $\mathcal{F}_{\phi}(\theta, \phi)$ almost vanishes completely. However, for further values of $\phi, \mathcal{F}_{\phi}(\theta, \phi)$ increases gradually to reach its maximum values at $\phi=\pi$. This behavior is repeated again at larger values of $\phi$.

Figs. $(5 a \& 5 b)$ are devoted to investigate the behavior of $\mathcal{F}_{\phi}(\theta, \phi)$ at a fixed value of $r=\pi / 8$ for a system is initially prepared in the singlet state, $\rho_{\psi^{-}}$. In Fig.(5a), the general behavior shows that, the Fisher information, $\mathcal{F}_{\phi}(\theta, \phi)$ increases gradually as $\theta$ increases to reach its maximum values at $\theta=\pi / 2$. At the same time as $\phi$ increases the effect of the weight parameter decreases gradually to vanish completely at $\phi \simeq \pi / 4$. The upper bounds of $\mathcal{F}_{\phi}(\theta, \phi)$ are depicted at $\phi=\pi$. These maximum values decrease as $\theta$ increases to reach its minimum value at $\theta=\pi / 2$. Fig $(5 \mathrm{~b})$ shows clearly the values of $\theta$ and $\phi$ which maximize and minimize the Fisher information.

The dynamics of the Fisher information of a teleported state by using a communication accelerated channel (within a single mode approximation), initially prepared in the $X$-state 

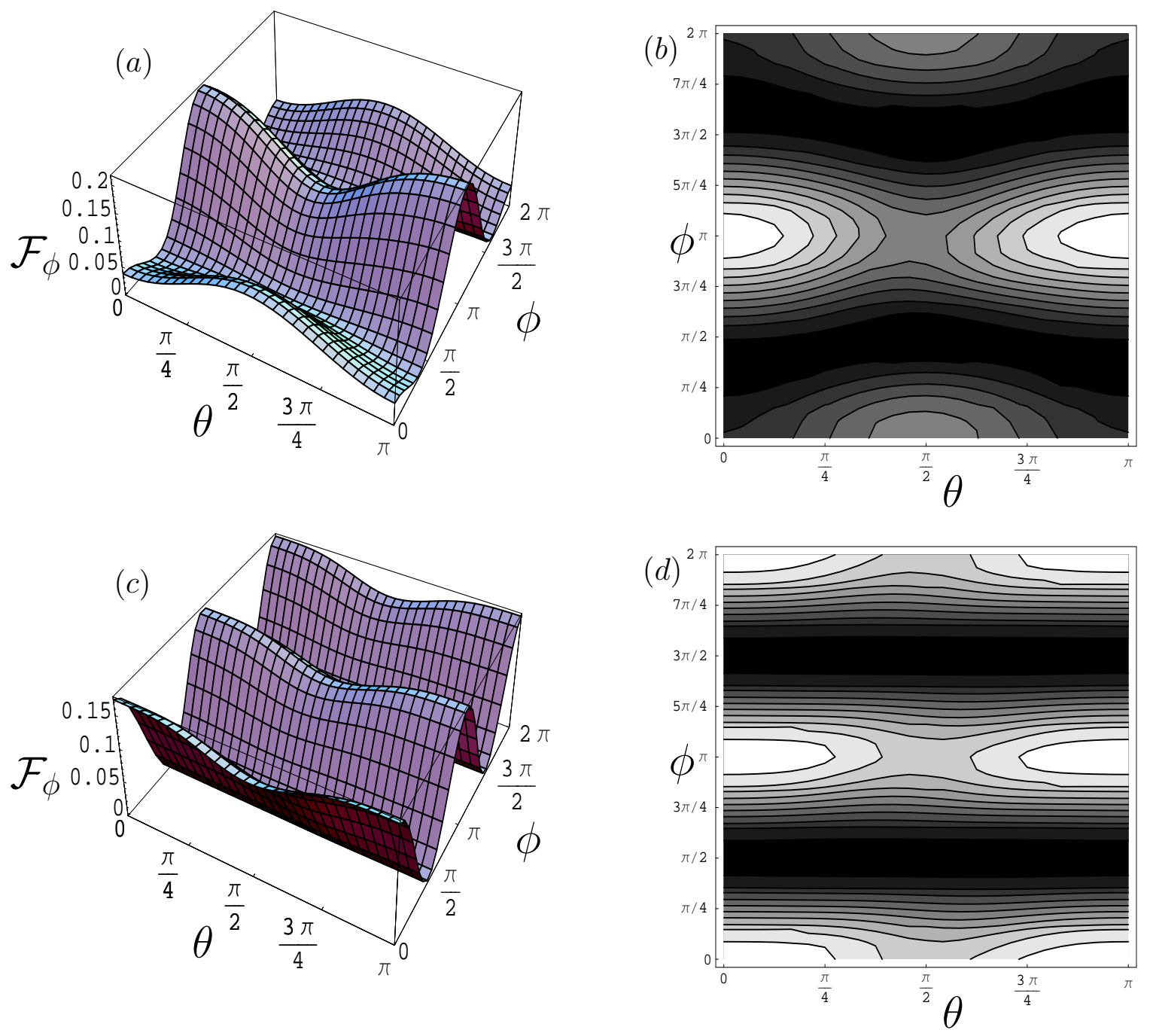

Figure 5: The dynamics of the Fisher information, $\mathcal{F}_{\phi}(\theta, \phi)$ at fixed $r=\pi / 8$, (a) BSMA for system is prepared initially in $\rho_{\psi^{-}}$, and ( b) WSMA for system is prepared initially in the $X$-state with $c_{1}=-0.9, c_{2}=-0.8, c_{3}=-0.7$.

is displayed in Figs. $(5 c \& 5 d)$. It is clear that, as $\theta$ increases, $\mathcal{F}_{\phi}(\theta, \phi)$ decays to reach its minimum value at $\theta=\pi / 2$. The effect of the weight parameter decreases as $\phi$ increases. On the other hand, the sudden changes of the Fisher information, $\mathcal{F}_{\phi}(\theta, \phi)$ are depicted as $\phi$ increases.

\subsection{Estimation of Unruh acceleration}

It is clear that, the final teleported state(8) not only depends on the initial parameter but also on the Unruh acceleration parameter. Therefore, it is important to estimate this parameter by quantifying the amount of Fisher information with respect to the Unruh acceleration, $\mathcal{F}_{r}(\theta, r)$. In Fig $(6)$, we investigate the effect of the initial parameters $\theta$ and $\phi$ on the behavior $\mathcal{F}_{r}(\theta, r)$ within/beyond the single mode approximation. The effect of the parameter $\theta$ is displayed in Figs. $(6 a \& 6 b)$, where we set $\phi=\pi / 4$. It is manifest that, $\mathcal{F}_{r}(\theta, r)$ increases as $r$ increases to reach its upper bounds at $\theta=0$. For further values of $\theta$, the Fisher information 

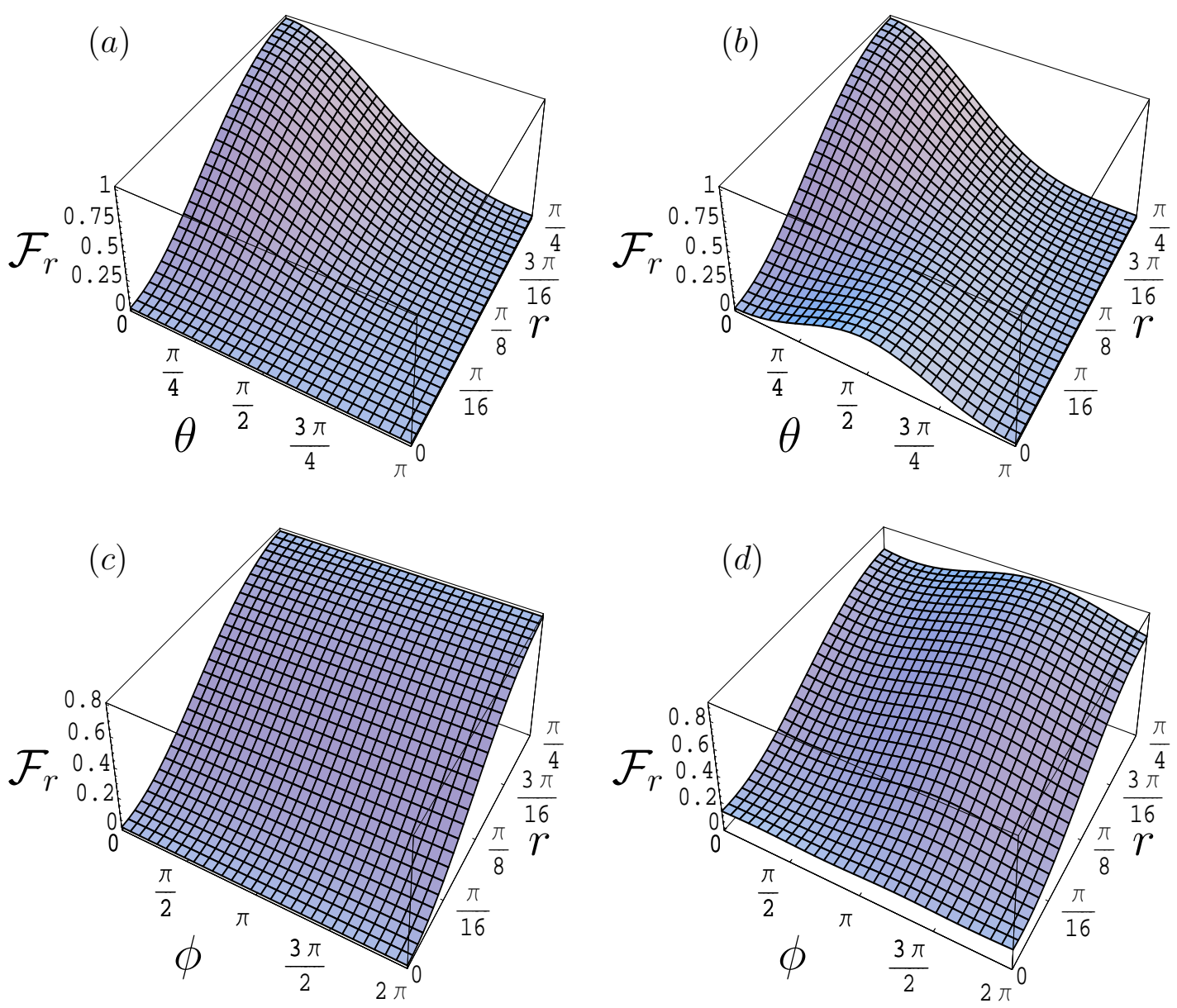

Figure 6: The Fisher information $\mathcal{F}_{r}(\theta, r)$, at fixed value of $\phi=\pi / 4$ for a system is initially prepared in the Bell state, $\rho_{\phi^{+}}$, for The figures (a) WSMA (b) BSMA. Figs(c,d) represent the Fisher information, $\mathcal{F}_{r}(\phi, r)$ at a fixed value of $\theta=\pi / 4$ where (c)WSMA and, (b) BSMA.

$\mathcal{F}_{r}$ decreases to vanish completely. For any arbitrary value of $\theta$ and small values of $r, \mathcal{F}_{r}$ is almost zero. However, this behavior is changed if the beyond single mode approximation is considered (Fig.(6b)), where $\mathcal{F}_{r}$ increases gradually as $\theta$ increases to reach its maximum values $\theta=\pi / 2$. For further values of $\theta$, Fisher information $\mathcal{F}_{r}$ decreases gradually to disappear at $\theta=\pi$.

The effect of the phase parameter on $\mathcal{F}_{r}(\phi, r)$ is described in Fig. $(6 c \& 6 d)$, where we set $\theta=\pi / 4$. It is clear that, within the single mode approximation, $\mathcal{F}_{r}(\phi, r)$ increases as $r$ increases, where the phase parameter has a feeble effect. On the other hand, beyond the single mode approximation $\mathcal{F}_{r}(\phi, r)$ increase gradually as $\phi$ increases and the maximum values are reached at $\phi=3 \pi / 2$. The upper bounds of Fisher information that depicted in Fig. $(5 c \& 5 c)$ is smaller than that shown in Fig. $(5 a \& 5 b)$.

\section{Conclusion}

In this contribution, we investigate the possibility of estimating the initial teleported parameters and the gained parameters during the teleportation process. The partner, Alice and 
Bob, share initially a communication channel of self-transposed class, which could be maximum entangled Bell state, or $X$-state. It is considered that, only Bob'squbit is accelerated while Alice's qubit is in the inertial frame. The final accelerated state between Alice and Bob is used to teleport unknown state from Alice to Bob by means of the teleportation protocol. The final teleported state depends on the initial parameters in addition to the Unruh acceleration parameter. Fisher information is used a measure of estimating the initial and the gained parameters, where we calculate it corresponding to each parameter within and beyond single mode approximations.

The possibility of estimating the teleported parameters within(beyond) the single mode approximation decreases/increases as the Unruh acceleration increases. The maximum values of estimation depend on the estimated parameter and the approximation mode. For estimating the teleported weight parameter at a particular value of the Unruh acceleration, the phase parameter has a slightly effect within the single mode approximation, while this effect is large beyond the single mode approximation. A similarly behavior is depicted when the teleported phase parameter is teleported, namely the weight parameter has a slight/large effect within/beyond single mode approximation, respectively.

Estimating the gained parameter,(Unruh acceleration) is discussed within/beyond the single mode approximation. It is clear that, the degree of estimation increases as the weight parameter increases to reach its maximum value, when the initial teleported state encode only classical information. For small values of acceleration, the weight parameter, the phase parameter have a slightly effect on the degree of estimating the acceleration parameter within the single mode approximation. The maximum value of estimating the gained parameter for arbitrary weight parameter is larger than that depicted for the arbitrary value of the phase parameter.

The effect of different cases of the initial states also, is discussed, where we show that using different classes of Bell states cause a shift of the maximum and minimum bounds of the Fisher information. Moreover, there are some extra tops appear for the singlet state. The degree of estimating of the teleported parameter by using $X$ - state is similar to that predicted for the singlet state but with smaller upper bounds, where the degree of estimation depends on the initial entanglement of the communication channel.

In conclusion, it is possible to estimate the teleported and the gained parameters by means of Fisher information. The maximum values of the estimation degree depend on the used approximation, the entanglement of the initial state between the partners and the structure of the initial teleported state. One can estimate these parameters with a large probability if the users teleported a classical information.

\section{References}

[1] A. S. Holevo" Probabilistic and statistical Aspects of Quantum Theory", (North Holland, Amsterdam 1982).

[2] Jian Ma and Xiaoguang Wang, " Fisher information and spin squeezing in the LipkinMeshkov-Glick model”,Phys. Rev. A 80, 012318 (2009).

[3] S. L. Braunstein and C. M. Caves," Statistical distance and the geometry of quantum states ", Phys. Rev. Lett. 72, 3439 (1994). 
[4] L.Ruppert, D. Virosztek and K. Hangos," Optimal parameter estimation of Pauli channels", J. Phys. A: Math. Theor. 45265305 (14pp)(2012).

[5] Q. Zheng, Y. TYao, amd Y. Li," Optimal quantum channel estimation of two interacting qubits subject to decoherece" Eur. Phys. J. D. 68170 (2014).

[6] P. Yue, Li. Ge and Q. Zheng," Invertible condition of quantum Fisher information matrix of a mixed qubit", Eur. Phys. J. D. 708 (2016).

[7] J. Ma, Yi.-X. Huang, X. Wang, and C. P. Sun," Quantum Fisher information of the greenberger-Horne- Zeilinger state in decoherence channel", Phys. Rev. . 84022302 (2011).

[8] J. Liu, H.-Na Xiong, F. Song, and X. Wang," Fidelity susceptibility and quantum Fisher or density operators with arbitrary ranks", Physica A410 167 (2014).

[9] H.-Na Xiong and X. Wang," Dynamical quantum Fisher information in the Ising model",Phsica A390 4719 (2011).

[10] A.Altinats," Quantum Fisher information of an open and noisy system in the steady state", Annals of Phys. 367192 (2016).

[11] J.He, Z.-Y. Ding and L.Ye," Enhancing quantum Fisher information by utlizing uncollapsing measurements", Physica A 457598 (2016).

[12] X. Xiao, Y. Yao, W.-J. Zhong, Y.-L.Li, and Y.-M. Xie," Enhancing teleportation of quantum Fisher information by partial measurements", Phys. Rev. A 93012307 (2016).

[13] Y. Yao, X. Xiao, LiGe, X.-F. Wang, and C.-pu Sun," Quantum Fisher information in noninertial frames", Phys. Rev. A 89042336 (2014).

[14] S. Banerjee, A. k. Alok, and S. Omkar," Quantum Fisher information and Skew information for Unruh accelerated Dirac qubit", Eur. Phys. J. C 76437 (2016).

[15] N. Metwally,"Unruh acceleration effect on the precision of parameter estimation", arXiv:1609.02092 (2016).

[16] C.H. Bennett, G. Brassard, S. Popescu, B. Schumacher, J.A. Smolin, W.K. Wootters," Purification of Noisy Entanglement and Faithful Teleportation via Noisy Channels", Phys. Rev. Lett. 76722 (1996).

[17] D. Deutsch, A. Ekert, R. Jozsa, C. Macchiavello, S. Popescu, A. Sanpera," Quantum Privacy Amplification and the Security of Quantum Cryptography over Noisy Channels", Phys. Rev. Lett. 772818 (1996).

[18] B.-G. Englert and N. Metwally,"Separability of entangled q-bit pairs", J. Mod. Opt. 47, pp.2221-2231 (2000).

[19] N. Metwally, "Usefulness classes of travelling entangled channels in nonintertial frames" Int. J. Mod Phys. B, Vol. 27281350155 (2013).

[20] P. M. Alsing, I. F. Schuller, R. B. Mann and T. E. Tessier, "Entanglement of Dirac fields in noninertial frames," Phys. Rev. A 74 pp. 032326-032340 (2006). 
[21] E. M. Martinez, I. Fuentes,"Redistribution of particle and antiparticle entanglement in noninertial frames", Phys. Rev. A83, pp. 052306-0523314 (2011).

[22] D. E. Bruschi, J. Louko, E. Martn-Martnez, A. Dragan, and I. Fuentes," Unruh effect in quantum information beyond the single-mode approximation," Phys. Rev. A 82 pp. 042332-042342 (2010).

[23] W.Zhong, Z. Sun, J. Ma, X. Wang and F. Nori,"Fisher Information under decoherence in Bloch representation" Phys. rev. A. 87022337 (2013). 